\title{
Melatonin Modulates Lipid Metabolism in Porcine Cumulus-Oocyte Complex via Its Receptors
}

Tianqi Zhu, Shengyu Guan, Dongying Lv, Mengmeng Zhao, Laiqing Yan, Li Shi, Pengyun Ji, Lu Zhang* and Guoshi Liu*

Key Laboratory of Animal Genetics, Breeding and Reproduction, National Engineering Laboratory for Animal Breeding, Key Laboratory of Animal Genetics Improvement, Ministry of Agriculture, College of Animal Science and Technology, China Agricultural University, Beijing, China

OPEN ACCESS

Edited by:

Shao-Chen Sun,

Nanjing Agricultural University, China

Reviewed by:

Guoquan Wu,

Yunnan Animal Science and Veterinary Institute, China

Xu Zhou,

Jilin University, China

Chunjin Li,

Jilin University, China

Zubing Cao,

Anhui Agricultural University, China

*Correspondence:

Lu Zhang

luzhang2018@cau.edu.cn

Guoshi Liu

gshliu@cau.edu.cn

Specialty section:

This article was submitted to Cell Growth and Division,

a section of the journa

Frontiers in Cell and Developmental

Biology

Received: 31 December 2020

Accepted: 18 February 2021

Published: 01 April 2021

Citation:

Zhu T, Guan S, Lv D, Zhao M, Yan L, Shi L, Ji P, Zhang L and Liu G (2021) Melatonin Modulates Lipid Metabolism in Porcine Cumulus-Oocyte Complex via Its Receptors. Front. Cell Dev. Biol. 9:648209. doi: $10.3389 / f c e l l .2021 .648209$
Lipid is a crucial energy resource for mammalian oocyte. Melatonin could benefit the maturation of porcine oocyte in vitro, but the related mechanism is not elucidated yet. In the current study, methods to monitor lipid metabolism in single live oocytes were firstly established using probes (Lipi-Blue and Lipi-Green). It was observed that both lipid biogenesis and lipolysis occurred in maturing oocyte, but the general level of lipids dropped. Then maturing oocytes stained with probes were treated with melatonin or lipid metabolic-related inhibitors (triacsin C, rotenone, or etomoxir). The results showed that the lipid metabolism and maturation of porcine oocytes were all disrupted and that melatonin rescued the oocytes treated with triacsin $\mathrm{C}$ or rotenone, but not those treated with etomoxir. Further investigation demonstrated that cumulus cells are able to transfer lipids to oocytes via gap junctions. It was also observed that melatonin receptors exist in cumulus cells and are required for oocytes to maintain lipid metabolism. Meanwhile, the global gene expressing in cumulus cells was also modulated by melatonin, especially the genes related to antioxidants (SOD1, GPX1, GPX3, GPX4, PRDX2, and PRDX5), lipid metabolism (FABP3, FABP5, ACACB, TECR, etc.), and mitochondrial respiration (GPD1, ETFB, CYC1, and the genes of ATP synthase). Altogether the current research demonstrates that melatonin modulates lipid metabolism in maturing oocytes through its receptors in cumulus cells and benefits the developmental competence of oocytes.

Keywords: melatonin, cumulus cell, oocyte, lipid metabolism, melatonin receptor

\section{INTRODUCTION}

During its growth phase, as the size of an oocyte increases obviously, a large amount of mRNAs, proteins, and lipids is accumulated in the oocyte cytoplasm. Shortly before ovulation, oocytes progress to meiotic resumption to accomplish the final nuclear and cytoplasmic maturation. These dynamic activities in oocytes consume enormous energy produced from various substrates stored

Abbreviations: LDs, Lipid droplets; CPT, Carnitine palmitoyl transferase; ACSLs, Long-chain acyl-coa synthetases; MT1, Melatonin receptor 1; MT2, Melatonin receptor 2; mtDNA, Mitochondrial DNA; DNMT1, DNA methyltransferase 1; COCs, Cumulus-oocytes complexes; ETC, Electron transport chain; DEGs, Differential expression genes; FRKM, Fragments per kilobase of exon per million mapped reads; PCA, Principle component analysis; GV, Germinal vesicle; GVBD, Germinal vesicle breakdown; FAO, Fatty acid oxidation; CBX, Carbenoxolone; ROS, Reactive oxygen species. 
in the oocyte or absorbed from the surroundings, such as glucose, pyruvate, lipids, and amino acids (Dunning et al., 2014). Oocytes that matured in the artificial medium described showed altered metabolism, resulting in lower developmental competence (Bradley and Swann, 2019). Therefore, well-balanced energy substrate provision is definitely required for oocyte maturation and further embryo development, which is critical for both assisted reproductive technology in a clinical setting and embryo production in the livestock industry.

Both glucose and pyruvate can be utilized by porcine oocytes to generate energy for the resumption of meiosis and the acquisition of developmental competence (Lowe et al., 2019). Recently, attention has been placed on the involvement of lipid metabolism in oocyte maturation and preimplantation embryo development. The amount of endogenous lipids in porcine oocytes is exceptionally high compared with that in oocytes of other domestic animals (McEvoy et al., 2000). The reason for such a large amount of intracellular lipid in porcine oocytes and its contribution to energy provision during oocyte maturation and embryo development is still poorly understood.

Porcine oocyte contains about $161 \mathrm{ng}$ lipids, which are much higher than those in mouse (4 ng), sheep (89 ng), and bovine (63 ng) oocytes (McEvoy et al., 2000). Lipids are mainly stored in lipid droplets (LDs), which make porcine oocytes very dark. About $60 \%$ of total lipids in porcine oocyte are triglycerides (Sturmey and Leese, 2003). It has been shown that the level of triglycerides decreases as bovine and porcine oocytes progress through meiotic maturation (Ferguson and Leese, 1999; Sturmey and Leese, 2003; Romek et al., 2011), and this process coincides with increased lipolysis (Cetica et al., 2002). The fatty acids (FAs) in mammalian oocytes are cleaved from triglycerides stored in LDs and can be directly transported across the mitochondrial membrane by carnitine palmitoyltransferase (CPT) to be oxidized via $\beta$-oxidation (Dunning et al., 2010). The inhibition of CPT in mammalian oocytes by etomoxir (250 $\mu \mathrm{M}$ for mouse, $100 \mu \mathrm{M}$ for bovine, and $10 \mu \mathrm{M}$ for porcine) compromises embryo development post fertilization (Paczkowski et al., 2013). Meanwhile, the removal of LDs from mouse metaphase II (MII) oocytes could still develop normally in the blastocyst after in vitro fertilization. But blocking the synthesis of lipids by triacsin C, inhibitor of long-chain acyl-CoA synthetases (ACSLs), leads to severe impairment of mouse early embryonic development (Aizawa et al., 2019). Recently, it has been increasingly recognized that lipogenesis and lipolysis are essential for oocyte maturation and embryo development. But the proper status of lipid metabolism in mammalian oocytes is still undetermined because there is no method to monitor and quantify the lipid level in real time.

The beneficial effects of melatonin on the oocyte, sperm, and embryo of animals have been well recognized. It has been determined that melatonin enhances porcine oocyte maturation and embryo development. Firstly, there are about $10^{-11} \mathrm{M}$ melatonin that exist in the follicular fluid of the pig ovary (Shi et al., 2009). It was observed that melatonin appearing in the culture medium could promote oocyte quality and developmental competence (Kang et al., 2009; Shi et al., 2009). The expression of the melatonin receptor 1 (MT1) gene was identified in cumulus and granulosa cells, but not in oocytes (Kang et al., 2009). Meanwhile, numerous researches had been conducted to investigate the role of melatonin on mammalian reproductive activities. The results demonstrated that melatonin could protect porcine oocytes from defects induced by heat stress (Li et al., 2015, 2016) or chemical regents such as rotenone, malathion, aflatoxin $\mathrm{B} 1$, bisphenol $\mathrm{A}$, benzo(a)pyrene, and MEHP during its maturation in vitro (Miao et al., 2018; Park et al., 2018; Zhang et al., 2018; Cheng et al., 2019; Chen et al., 2020; Niu et al., 2020). Melatonin can also enhance mitochondrial biogenesis and reduce endoplasmic reticulum stress during in vitro maturation (IVM). Up to now, it is still undetermined whether the beneficial effect of melatonin on porcine oocyte maturation is mediated by its receptors or its direct anti-oxidation capability.

Recently, it has been demonstrated that melatonin regulates lipid metabolism in porcine oocytes, including lipogenesis, lipolysis, and mitochondrial biogenesis. Melatonin treatment significantly elevated the number of LDs and upregulated gene expression related to lipogenesis (Jin et al., 2017). Oocytes treated with melatonin formed smaller LDs and abundantly expressed several genes associated with lipolysis (Jin et al., 2017). Moreover, melatonin significantly increased the content of FAs, mitochondrial numbers, and ATP as indicated by fluorescent staining (Jin et al., 2017). Concomitantly, melatonin treatment upregulated gene expression related to FA $\beta$-oxidation and mitochondrial biogenesis (Jin et al., 2017). These results indicated that melatonin promoted lipid metabolism and thereby provided an energy source for oocyte maturation and subsequent embryonic development. However, another study showed that melatonin added to an IVM medium enhances LD accumulation as well as triglyceride level in porcine oocytes (He et al., 2018). Melatonin supplementation decreased mitochondrial membrane potential, mitochondrial respiratory chain complex IV activity, and mitochondrial reactive oxygen species in maturing oocytes, which indicated that melatonin suppresses mitochondrial activities in porcine oocytes ( $\mathrm{He}$ et al., 2018). Further investigation found that melatonin did not alter the copy number of mitochondrial DNA (mtDNA) but reduced the expression of mtDNA-encoded genes, which is mediated by DNMT1 (He et al., 2018). These results suggest that melatonin promotes oocyte maturation by inducing mitochondrial quiescence to reduce mROS production and enhance LD accumulation in porcine oocytes (He et al., 2018).

The evidence from the above studies demonstrated that melatonin promotes porcine oocyte maturation by regulating lipid metabolism. But it is still unrevealed whether melatonin affects lipid biogenesis or lipolysis in porcine oocytes. In the current study, the methods for monitoring lipid dynamics in maturing porcine oocytes were established using specific probes. Then the regulation of melatonin on lipid metabolism in porcine oocytes was evaluated. Meanwhile, the role of cumulus cells and their melatonin receptors in modulating lipid contents in oocyte was also evaluated by inhibiting gap junctions and melatonin receptors. The current results showed that melatonin enhances porcine oocyte maturation by regulating lipid transfer from cumulus cells to oocytes via melatonin receptors. 


\section{MATERIALS AND METHODS}

\section{Ethics Statement}

All the procedures for animal manipulations were performed according to the guidelines of the Animal Care and Use Committee of China Agricultural University and approved by the Ethics Committee of the Agriculture University of China (permission number: CAU20150915-1 SYXK).

\section{Chemicals}

All chemicals used in this study were purchased from the SigmaAldrich Chemical Company (St. Louis, MO, United States) unless otherwise indicated.

\section{Oocyte IVM}

Ovaries collected from pre-pubertal gilts were donated by a local slaughterhouse (Beijing Food Company, Beijing, China). Follicular fluid was aspirated from follicles $(3-6 \mathrm{~mm}$ in diameter) using a syringe and a $20-\mathrm{G}$ hypodermic needle. The cumulusoocyte complexes (COCs) were rinsed twice in HEPES-buffered Tyrode's Lactate (TL-HEPES) medium and then three times in maturation medium without hormones. The COCs were then transferred into the maturation medium (50 oocytes per $0.5 \mathrm{ml}$ of medium) consisting of TCM-199 supplemented with $0.57 \mathrm{mM}$ cysteine, $3.05 \mathrm{mM}$ D-glucose, $0.91 \mathrm{mM}$ sodium pyruvate, $10 \mathrm{ng} / \mathrm{ml}$ epidermal growth factor (EGF), $0.5 \mathrm{IU} / \mathrm{ml}$ ovine luteinizing hormone (LH), $0.5 \mathrm{IU} / \mathrm{ml}$ porcine folliclestimulating hormone (FSH), $0.1 \%$ polyvinyl alcohol (PVA), $75 \mathrm{mg} / \mathrm{ml}$ penicillin, $50 \mathrm{mg} / \mathrm{ml}$ streptomycin, $20 \mathrm{ng} / \mathrm{ml}$ LIF (EMD Millipore, MA, United States), $20 \mathrm{ng} / \mathrm{ml}$ IGF1 (ProSpec, Ness Ziona, Israel), and $40 \mathrm{ng} / \mathrm{ml} \mathrm{FGF2} \mathrm{(PeproTech,} \mathrm{NJ,} \mathrm{United} \mathrm{States)}$ (Abeydeera et al., 1998; Yuan et al., 2017). Meanwhile, the inhibitors or dyes applied to study lipid metabolism were added to the maturation medium as described in each paragraph. Three inhibitors were used to study lipid metabolism as described in the literature. Triacsin C served as an inhibitor of ACSLs, could block the synthesis of lipids, and could induce severe impairment of mouse early embryonic development (Aizawa et al., 2019). Etomoxir could irreversibly inhibit FA oxidation (FAO) by blocking CPT-1a (Lowe et al., 2019). Rotenone could disrupt the mitochondrial electron transport chain (ETC) in maturing oocytes through the inhibition of the ETC complex I, resulting in ATP depletion, mROS production, and damage of mitochondrial membrane potential (Niu et al., 2020). Maturation was conducted at $38.5^{\circ} \mathrm{C}$ and $5 \% \mathrm{CO}_{2}$ in air with $100 \%$ humidity. After $42-$ $44 \mathrm{~h}$ of maturation, the COCs were transferred into TL-HEPES containing $1 \mathrm{mg} / \mathrm{ml}$ hyaluronidase, and the cumulus cells were removed by vortex. The oocytes and cumulus cells were rinsed with TL-HEPES and then used for subsequent experiments.

\section{Parthenogenetic Activation of Oocytes}

The denuded porcine oocytes were activated in the activation medium [0.3 m mannitol, $0.05 \mathrm{~mm} \mathrm{CaCl}, 0.1 \mathrm{~mm} \mathrm{MgCl}$, and $0.1 \%$ bovine serum albumin (BSA)] by an electrical pulse of DC $130 \mathrm{~V} / \mathrm{mm}$ for $80 \mu$ s using a BTX Electro-Cell Manipulator 2001 (BTX, Inc., San Diego, CA, United States). The oocytes were then rinsed in porcine zygote medium-3 (PZM-3) and cultured in the medium containing $5 \mu \mathrm{g} / \mathrm{ml}$ of cytochalasin B for $5-6 \mathrm{~h}$, at $38.5^{\circ} \mathrm{C}$ and $5 \% \mathrm{CO}_{2}$ in air with $100 \%$ humidity. Subsequently, oocytes were rinsed in PZM supplemented with $0.6 \mathrm{mg} / \mathrm{ml}$ of FA-free BSA for embryo culture.

\section{In vitro Culture (IVC) of Embryos}

Parthenogenetically activated oocytes, in groups of approximately 15-20, were cultured in $100 \mu$ l droplets of PZM-3 supplemented with $0.6 \mathrm{mg} / \mathrm{ml}$ of BSA. Melatonin stock dissolved in TCM-199 was added to the culture medium according to the experimental design. The oocytes were then cultured in the medium at $39^{\circ} \mathrm{C}, 5 \% \mathrm{CO}_{2}$, and $5 \% \mathrm{O}_{2}$. The cleavage rate and blastocyst rate were observed and recorded at 48 and 168 h of IVC, respectively.

\section{Observation of LDs in Porcine and Mouse Oocytes}

The COCs were placed in the TL-HEPES medium containing $0.1 \mu \mathrm{mol} / \mathrm{L}$ Lipi-Blue (Dojindo Laboratories, Kumamoto, Japan) for $30 \mathrm{~min}$ and then incubated in maturation medium. At 18 and $44 \mathrm{~h}$ of maturation, the COCs were taken out from the IVM medium to be stained by Lipi-Green (Dojindo Laboratories, Kumamoto, Japan). The cumulus cells surrounding oocytes were removed, and then the MII oocytes were placed in a glass petri dish for imaging using a confocal microscope (Nikon A1 HD25, Tokyo, Japan) with excitation wavelength $\lambda$ ex $=405 \mathrm{~nm}$ for LipiBlue and $\lambda e x=488 \mathrm{~nm}$ for Lipi-Green. ImageJ (Version 1.53, National Institutes of Health, United States) was used to process the images and record the fluorescence intensities of LDs.

Mouse oocytes from melatonin receptor-knockout (Mtnr1a $a^{-/-}$and $M t n r 1 b^{-/-}$) or wild-type mice were collected as described in our previous research (Zhang et al., 2019). Briefly, at the age of 8 weeks, the mice were injected with 5 IU PMSG, 46-48 h later, followed by 5 IU hCG to induce superovulation. Thirteen to fifteen hours after hCG injection, COCs at MII stage were isolated from female mice's ampullas. Then denuded MII oocytes were stained with $0.1 \mu \mathrm{mol} / \mathrm{L}$ of Lipi-Blue for $30 \mathrm{~min}$, and then the mouse oocytes were placed in a glass petri dish for imaging with a confocal microscope.

\section{Lipid Analysis by UPLC-MS/MS Lipid Extraction}

Denuded MII oocytes were washed three times with phosphatebuffered saline (PBS) solution and stored in microtubes at $-80^{\circ} \mathrm{C}$ until analysis. The extraction was performed according to the protocol described by Mateus et al. (2016) with modifications (Sudano et al., 2016). Four hundred oocytes per sample were counted, and the metabolites were extracted using a solution of $400 \mu \mathrm{l}$ methanol:water $(4: 1, \mathrm{v} / \mathrm{v})$. The mixture was settled at $-20^{\circ} \mathrm{C}$ and treated by a high-throughput tissue crusher Wonbio96c (Shanghai Wanbo Biotechnology Co., Ltd.) at $50 \mathrm{~Hz}$ for $6 \mathrm{~min}$; then the samples were placed at $-20^{\circ} \mathrm{C}$ for $30 \mathrm{~min}$ to precipitate proteins. After centrifugation at $13,000 \mathrm{~g}$ at $4^{\circ} \mathrm{C}$ for $15 \mathrm{~min}$, the supernatant was carefully transferred to sample vials for LC-MS/MS analysis. 


\section{UPLC-MS/MS Analysis}

Chromatographic separation of the metabolites was performed on an ExionLC ${ }^{\mathrm{TM}} \mathrm{AD}$ system (AB Sciex, United States) equipped with an ACQUITY UPLC BEH C18 column $(100 \mathrm{~mm} \times 2.1 \mathrm{~mm}$ i.d., $1.7 \mu \mathrm{m}$; Waters, Milford, United States). The mobile phases consisted of $0.1 \%$ formic acid in water (solvent $\mathrm{A}$ ) and $0.1 \%$ formic acid in acetonitrile:isopropanol $(1: 1, \mathrm{v} / \mathrm{v})$ (solvent $\mathrm{B})$. The solvent gradient changed according to the following conditions: from 0 to $3 \mathrm{~min}, 95 \%$ (A):5\% (B) to $80 \%(\mathrm{~A}): 20 \%$ (B); from 3 to $9 \mathrm{~min}, 80 \%(\mathrm{~A}): 20 \%(\mathrm{~B})$ to $5 \%(\mathrm{~A}): 95 \%(\mathrm{~B})$; from 9 to $13 \mathrm{~min}, 5 \%(\mathrm{~A}): 95 \%$ (B) to $5 \%(\mathrm{~A}): 95 \%(\mathrm{~B})$; from 13 to $13.1 \mathrm{~min}, 5 \%(\mathrm{~A}): 95 \%(\mathrm{~B})$ to $95 \%(\mathrm{~A}): 5 \%(\mathrm{~B})$; and from 13.1 to $16 \mathrm{~min}, 95 \%(\mathrm{~A}): 5 \%(\mathrm{~B})$ to $95 \%(\mathrm{~A}): 5 \%$ (B) to equilibrate the systems. The column temperature was maintained at $40^{\circ} \mathrm{C}$. The sample injection volume was $20 \mu \mathrm{l}$, and the flow rate was set to $0.4 \mathrm{ml} / \mathrm{min}$. The UPLC system was coupled to a quadrupoletime-of-flight mass spectrometer (Triple $\mathrm{TOF}^{\mathrm{TM}} 5600^{+}$, $\mathrm{AB}$ Sciex, United States) equipped with an electrospray ionization (ESI) source operating in positive and negative modes. Data acquisition was performed with the data-dependent acquisition (DDA) mode. The detection was carried out over a mass range of $50-1,000 \mathrm{~m} / \mathrm{z}$.

\section{Data Preprocessing and Annotation}

After UPLC-TOF/MS analyses, the raw data were processed by Progenesis QI 2.3 (Non-linear Dynamics, Waters, United States) for peak detection and alignment. Metabolic features that detected at least $80 \%$ in any set of samples were retained. Mass spectra of the metabolic features were identified by using the accurate mass, MS/MS fragments spectra, and isotope ratio difference by searching in reliable biochemical databases such as the Human Metabolome Database (HMDB) ${ }^{1}$ and METLIN database $^{2}$. Concretely, the mass tolerance between the measured $\mathrm{m} / z$ values and the exact mass of the components of interest was $\pm 10 \mathrm{ppm}$. For metabolites having MS/MS confirmation, only the ones with an MS/MS fragment score above 30 were considered as confidently identified. Otherwise, metabolites had only tentative assignments.

\section{Transcriptomic Analysis of Cumulus Cells \\ RNA Extraction}

The cumulus cells were collected from COCs $(n=300$ oocytes, six replicates), and then the total RNA was extracted from the tissue using the TRIzol reagent according to the manufacturer's instructions (Invitrogen), and genomic DNA was removed using DNase I (Takara). Then RNA quality was determined by the 2100 Bioanalyzer (Agilent) and quantified using the ND2000 (NanoDrop Technologies). Only samples with high-quality RNA $(\mathrm{OD} 260 / 280=1.8-2.2, \mathrm{OD} 260 / 230 \geq 2.0, \mathrm{RIN} \geq 6.5$, $28 \mathrm{~S}: 18 \mathrm{~S} \geq 1.0,>2 \mu \mathrm{g})$ were selected to construct the sequencing library.

\footnotetext{
${ }^{1}$ http://www.hmdb.ca/

${ }^{2}$ https://metlin.scripps.edu/
}

\section{Library Preparation and Sequencing}

The RNA-seq transcriptome library was prepared following a TruSeq $^{\text {TM }}$ RNA sample preparation kit from Illumina (San Diego, CA) using $1 \mu \mathrm{g}$ of total RNA. Libraries were selected for cDNA target fragments of 200-300 bp on 2\% low-range ultra-agarose followed by PCR amplified using Phusion DNA polymerase (NEB) for 15 PCR cycles. After being quantified by TBS380, the paired-end RNA-seq sequencing library was sequenced with the Illumina HiSeq X Ten/NovaSeq 6000 sequencer (San Diego, CA).

\section{Differential Expression Analysis and Functional Enrichment}

The raw paired-end reads were trimmed and quality controlled by SeqPrep ${ }^{3}$ and Sickle ${ }^{4}$ with default parameters. Then clean reads were separately aligned to the reference genome ${ }^{5}$ with an orientation mode using TopHat $^{6}$ (version 2.0.0) software (Trapnell et al., 2009).

To identify differentially expressed genes (DEGs) between two different samples, the expression level of each transcript was calculated according to the fragments per kilobase of exon per million mapped reads (FRKM) method. RSEM $^{7}$ was used to quantify gene abundances (Li and Dewey, 2011). R statistical package software $\operatorname{EdgeR}^{8}$ (Robinson et al., 2010) was utilized for differential expression analysis. In addition, functional enrichment analyses including GO and KEGG were performed to identify which DEGs were significantly enriched in GO terms and metabolic pathways at a Bonferroni-corrected $P<0.05$ compared with the whole-transcriptome background. GO functional enrichment and KEGG pathway analysis were carried out with Goatools ${ }^{9}$ and KOBAS ${ }^{10}$ (Xie et al., 2011).

\section{Gene Expression by Quantitative Real-Time PCR (RT-PCR)}

Cumulus cells were collected from COCs and then washed three times with $\mathrm{PBS}$ and stored at $-80^{\circ} \mathrm{C}$ until RNA extraction. Total RNA was extracted using TRIzol (Invitrogen Inc., Carlsbad, CA, United States) and quantified by measuring absorbance at $260 \mathrm{~nm}$ and stored at $-80^{\circ} \mathrm{C}$ until assay. The mRNA levels of relevant genes were evaluated by quantitative RT-PCR using the OneStep SYBR PrimeScript RT-PCR Kit (Takara Bio. Inc., Tokyo, Japan) in a LightCycler (Roche Applied Science, Mannheim, Germany). Accumulated levels of fluorescence were analyzed by the second derivative method after the melting curve analysis, and then the expression levels of target genes were normalized to the expression level of GAPDH in each sample. Primer pairs of analyzed mRNAs are described in Table 1.

\footnotetext{
${ }^{3}$ https://github.com/jstjohn/SeqPrep

${ }^{4}$ https://github.com/najoshi/sickle

${ }^{5}$ http://asia.ensembl.org/Sus_scrofa/Info/Index

${ }^{6}$ http://tophat.cbcb.umd.edu/

${ }^{7}$ http://deweylab.biostat.wisc.edu/rsem/

${ }^{8}$ http://www.bioconductor.org/packages/2.12/bioc/html/edgeR.html

${ }^{9}$ https://github.com/tanghaibao/Goatools

${ }^{10}$ http://kobas.cbi.pku.edu.cn/home.do
} 
TABLE 1 | The primers used for real-time q-PCR.

\begin{tabular}{|c|c|c|c|}
\hline \multirow{2}{*}{$\begin{array}{l}\text { Target genes } \\
\text { GAPDH }\end{array}$} & \multirow{2}{*}{$\begin{array}{l}\text { Accession number } \\
\text { NM_001206359.1 }\end{array}$} & \multicolumn{2}{|c|}{ Primer sequences $\left(5^{\prime}-3^{\prime}\right)$} \\
\hline & & $\mathrm{F}$ & GTCGGTTGTGGATCTGACCT \\
\hline & & $\mathrm{R}$ & GTCCTCAGTGTAGCCCAGGA \\
\hline \multirow[t]{2}{*}{ ACACA } & NM_001114269.1 & $\mathrm{F}$ & TाGTTACTCGTITGGTGGGA \\
\hline & & $\mathrm{R}$ & AGCGTTGGCTITCAGGTCTT \\
\hline \multirow[t]{2}{*}{ FASN } & NM_001099930.1 & $\mathrm{F}$ & TGTCCTGGGAAGAGTGTAAGCA \\
\hline & & $\mathrm{R}$ & GCAGGAACTCGGACATAGCG \\
\hline \multirow[t]{2}{*}{ PLIN2 } & NM_214200.2 & $\mathrm{F}$ & ATGGCTGGCGACATCTACTCA \\
\hline & & $\mathrm{R}$ & TGCCCCTTGCTGGAACTG \\
\hline \multirow[t]{2}{*}{ CGI-58 } & NM_001012407.1 & $\mathrm{F}$ & AGGAGGTCTCGGACTITGGG \\
\hline & & $\mathrm{R}$ & GGTCTGGTCGCTCAGGAAAA \\
\hline \multirow[t]{2}{*}{ PPAR $\gamma$} & NM_214379.1 & $\mathrm{F}$ & GCATCTITAGGGGTGTCAGTT \\
\hline & & $\mathrm{R}$ & CGTGGACGCCATACTITAGGA \\
\hline \multirow[t]{2}{*}{ СРТ2 } & NM_001246243.1 & $\mathrm{F}$ & TGTCCCAGTATITCGGCTाT \\
\hline & & $\mathrm{R}$ & GTCTCCTCGTTGCCACCCT \\
\hline \multirow[t]{2}{*}{ CPT1a } & NM_001129805.1 & $\mathrm{F}$ & TAAACGGATGACGGCTCTGG \\
\hline & & $\mathrm{R}$ & TGTGGGTCGGGGTGATGT \\
\hline \multirow[t]{2}{*}{ PGC-1 $1 \alpha$} & NM_213963.2 & $\mathrm{F}$ & AATCGCAGTCGCAACATITACA \\
\hline & & $\mathrm{R}$ & TGGGTCCCCGAAGACTCAC \\
\hline \multirow[t]{2}{*}{ TFAM } & NM_001130211.1 & $\mathrm{F}$ & GGACCTCTGTGCGGTITGTG \\
\hline & & $\mathrm{R}$ & CACCTGCCAGTCTGCCCTA \\
\hline \multirow[t]{2}{*}{ PRDX2 } & NM_001244474.1 & $\mathrm{F}$ & TGGGACGCTCTGTGGATGA \\
\hline & & $\mathrm{R}$ & GGGGCAGGTCTGGCTाT \\
\hline
\end{tabular}

\section{Statistical Analyses}

Unless specifically described, the rest of the data were expressed as mean \pm SEM. The significance of differences between mean values was analyzed by ANOVA, followed by Dunnett's post hoc test using SPSS 18.0 statistical software (SPSS Inc., Chicago, IL, United States). The significant difference between treatments was set to $P<0.05$.

For lipidomics analysis of porcine oocyte, multivariate statistical analysis was performed using the ropls $\mathrm{R}$ package (Version 1.6.2) ${ }^{11}$. Principal component analysis (PCA) using an unsupervised method was applied to obtain an overview of the metabolic data, general clustering, trends, or outliers, which were visualized. Variable importance (VIP) was calculated in the OPLS-DA model. $P$ values were estimated with paired Student's $t$-test on single-dimensional statistical analysis. Statistical significance among groups was considered as a VIP value more than 1 and $P<0.05$.

\section{RESULTS}

\section{Dynamics of LDs in Porcine Oocyte During IVM}

In order to detect the LDs in living oocytes, lipid-specific probes (Lipi-Blue and Lipi-Green) were tested in porcine oocytes at different maturation phases. As described by a previous study, both probes can be used to monitor the lipid changes in living cells for over $48 \mathrm{~h}$ (Tatenaka et al., 2019). It was observed

\footnotetext{
${ }^{11} \mathrm{http} / / /$ bioconductor.org/packages/release/bioc/html/ropls.html
}

that both blue and green fluorescence decreased as the oocyte grew from the GV to MII stages (Figure 1A). The oocytes were stained with Lipi-Blue at the GV stage, and then the images were acquired at the GV (210.52 \pm 4.03$)$, MI $(110.06 \pm 8.92)$, and MII $(106.87 \pm 7.34)$ stages, so the decrease of blue fluorescence intensity indicates the mobilization of lipids to generate energy for the activities of oocytes (Figure 1B). Meanwhile, the oocytes were co-incubated with Lipi-Green at the GV, MI, and MII stages; thus, the intensity of green fluorescence represents the total lipids contained in each time point, which are also reduced as oocytes grow (Figure 1C) from stages GV $(244.75 \pm 2.25)$ to MI $(211.26 \pm 9.85)$ to MII $(156.80 \pm 10.44)$. The ratio of LipiGreen/Lipi-Blue is also lower in the MII oocytes (Figure 1D) (GV: $1.645 \pm 0.25$, MI: $0.67 \pm 0.047$, and MII: $0.64 \pm 0.038$ ). These observations provide a method to monitor the dynamics of lipid in single living oocytes.

\section{Melatonin Modulates the Lipid Metabolism in Porcine Oocyte}

Agonists targeted at the pathway of lipid metabolism were applied to investigate how melatonin regulates lipid metabolism in maturing porcine oocytes (Figure 2). Compared with the control group $(139.11 \pm 6.58)$, melatonin-treated oocytes showed higher blue fluorescence intensity $(158.77 \pm 5.96)$, which indicates that less lipids stored in GV oocytes were mobilized. When the oocytes were treated with etomoxir, an irreversible inhibitor of FAO blocking CPT-1a (Lowe et al., 2019), the blue and green fluorescence intensities $(241.83 \pm 1.64)$ are all significantly higher than that in oocytes of the control group, and melatonin cannot reverse these results.

When the lipogenesis of oocytes was suppressed by triacsin $\mathrm{C}$, an inhibitor of ACSLs which can block the de novo synthesis of triglycerides (Aizawa et al., 2019), diglycerides, and cholesterol esters, the blue fluorescence intensity $(117.32 \pm 6.85)$ is significantly lower than that in oocytes of the control group, and melatonin treatment could reverse this result $(151.11 \pm 6.10)$. Similarly, the disruption of the mitochondrial ETC in maturing oocytes through the inhibition of complex I by rotenone (Won et al., 2015) leads to reduced blue fluorescence intensity $(103.85 \pm 10.26)$, which was reversed by melatonin $(137.39 \pm 8.43)$. Altogether, these results indicate that melatonin suppressed lipolysis to generate energy and promote lipid store in maturing porcine oocytes.

To detect the changes of lipid contents induced by melatonin, 400 oocytes were collected for each sample with seven repeats for both the melatonin and control groups and were used for the UPLC-MS/MS study. In total, 298 FAs were identified. As the results showed in Table 2, the levels of 13 lipids were altered by melatonin; among them, eight FAs were upregulated, and five FAs were downregulated.

\section{Lipid Metabolism Regulates the Developmental Competence of Porcine Oocytes}

To determine how melatonin affects the maturation of porcine oocytes through modulating the lipid metabolism, the maturing 


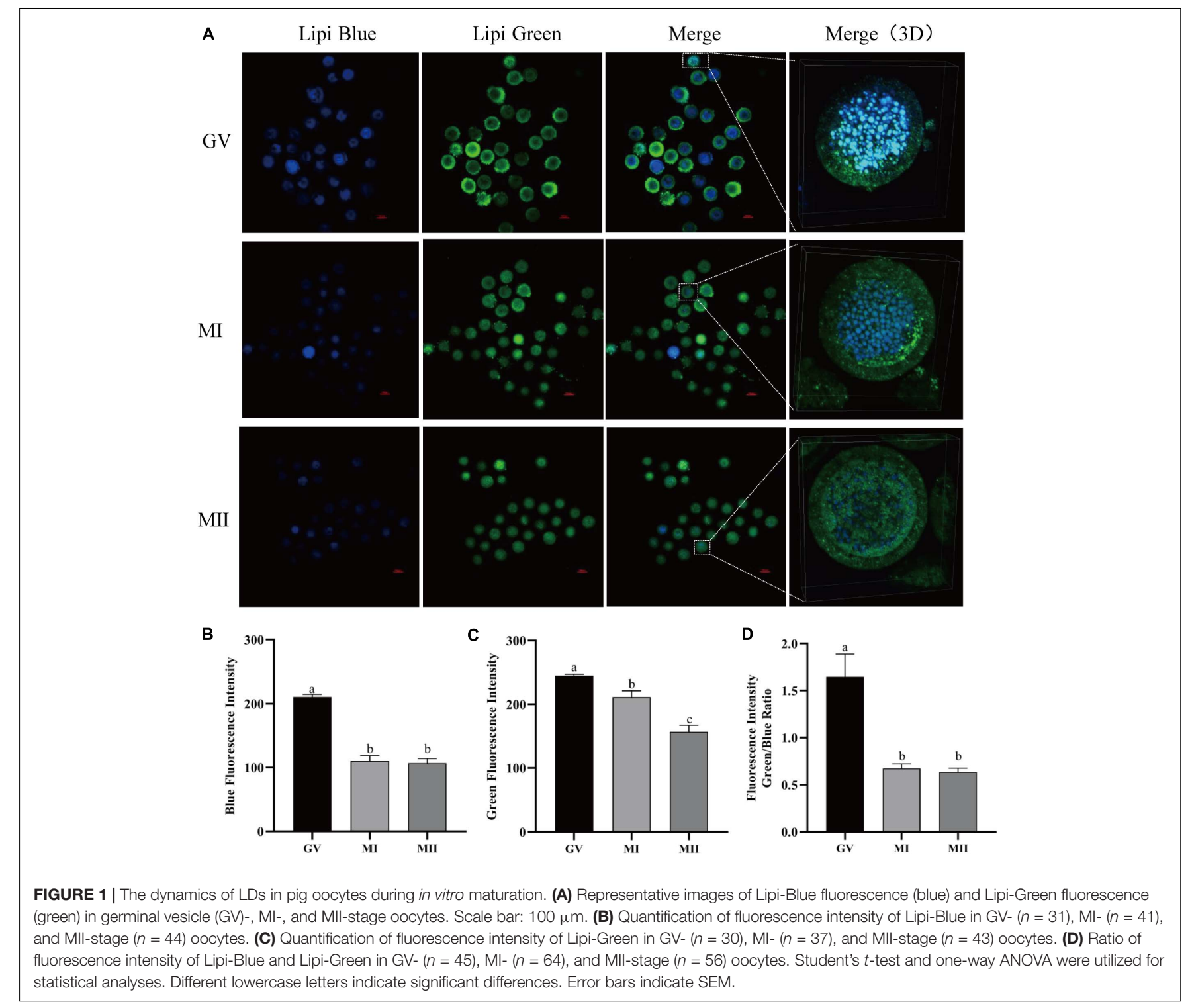

TABLE 2 | The fatty acid profiles of oocytes from the control and melatonin groups.

\begin{tabular}{|c|c|c|c|c|c|c|c|}
\hline Metabolites & Mz & Type & IonFormula & Class & Subclass & FC(MT/CON) & $P$-value \\
\hline$M G(20: 1 p)$ & 386.3629 & $M G(20: 1 p)+N H 4$ & $\mathrm{C} 23 \mathrm{H} 48 \mathrm{O} 3 \mathrm{~N} 1$ & $\mathrm{GL}$ & MG & 4.056503024 & 0.020551 \\
\hline PC(34:3) & 756.5538 & $P C(34: 3)+H$ & C42 H79 O8 N1 P1 & GP & PC & 1.7938854 & 0.041914 \\
\hline PC(38:5) & 808.5851 & $P C(38: 5)+H$ & C46 H83 O8 N1 P1 & GP & PC & 0.555480605 & 0.018347 \\
\hline PE(20:0p) & 530.3217 & $P E(20: 0 p)+\mathrm{Na}$ & C25 H50 O7 N1 P1 Na1 & GP & $\mathrm{PE}$ & 1.32368186 & 0.019753 \\
\hline TG(16:0/18:1/18:1) & 876.8015 & $\mathrm{TG}(16: 0 / 18: 1 / 18: 1)+\mathrm{NH} 4$ & $\mathrm{C} 55 \mathrm{H} 106 \mathrm{O} 6 \mathrm{~N} 1$ & GL & $\mathrm{TG}$ & 5.550013905 & 0.036007 \\
\hline LPE(22:3) & 530.3252 & LPE(22:3)-H & C27 H49 O7 N1 P1 & GP & LPE & 1.456611091 & 0.012236 \\
\hline LPE(22:4) & 528.3096 & LPE(22:4)-H & C27 H47 O7 N1 P1 & GP & LPE & 1.356583578 & 0.009065 \\
\hline $\operatorname{LPI}(18: 0)$ & 599.3202 & LPI(18:0)-H & C27 H52 O12 N0 P1 & GP & LPI & 1.859655426 & 0.038688 \\
\hline $\operatorname{SM}(d 36: 1)$ & 775.5971 & $\mathrm{SM}(\mathrm{d} 36: 1)+\mathrm{HCOO}$ & C42 H84 O8 N2 P1 & $\mathrm{SP}$ & SM & 0.616533814 & 0.025273 \\
\hline $\mathrm{PC}(37: 4)$ & 796.5851 & $\mathrm{PC}(37: 4)+\mathrm{H}$ & C45 H83 O8 N1 P1 & GP & PC & 0.195726296 & 0.022136 \\
\hline
\end{tabular}




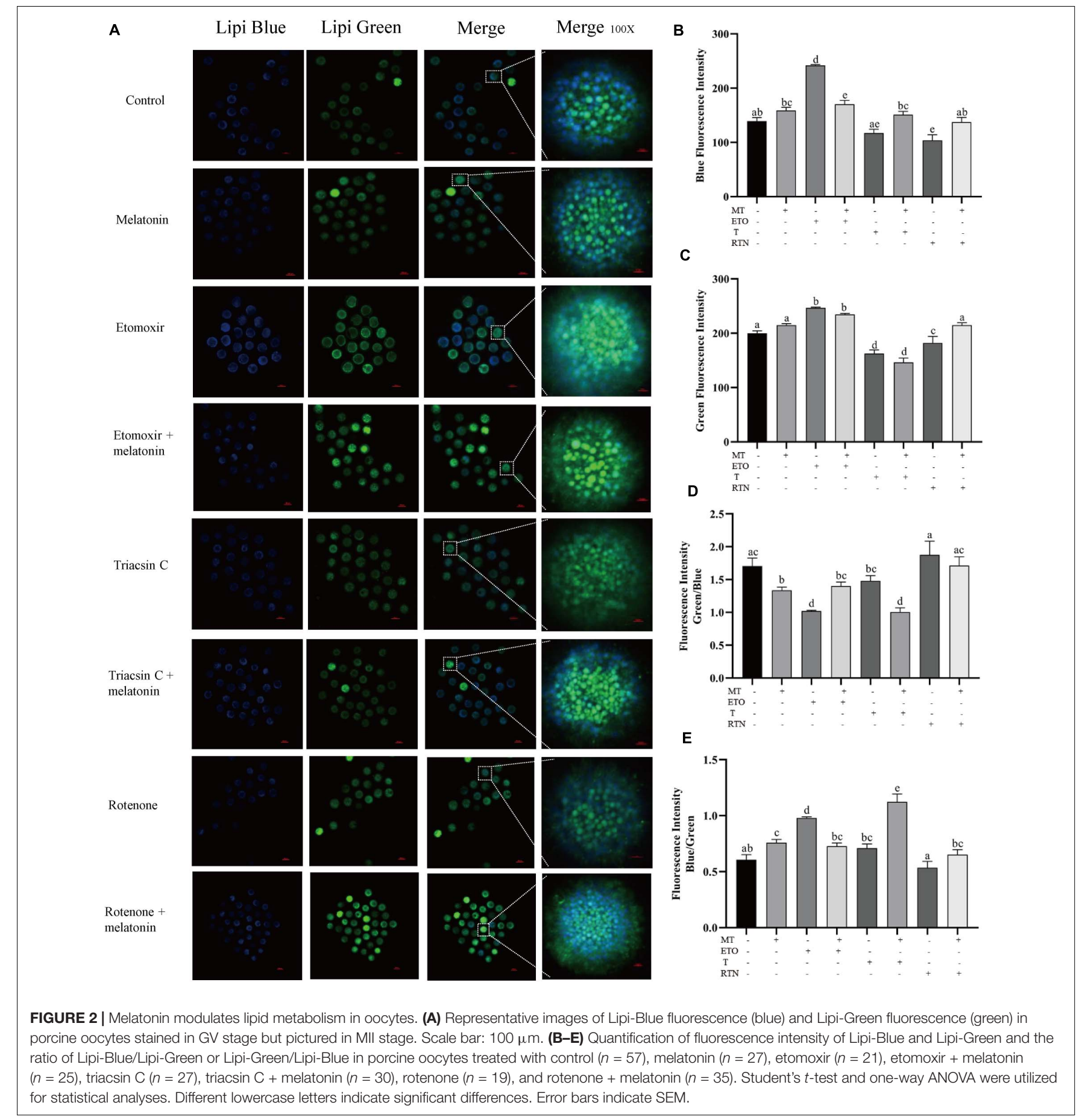

oocytes were treated with the agonists used above (Figure 3). Firstly, the supplementation of melatonin does not alter the first polar body extrusion $(71.63 \pm 2.26 \%$ vs. $72.94 \pm 2.475 \%)$, which represents nuclear maturation, but rescues the defects caused by triacsin C (56.19 $\pm 38.6 \%$ vs. $62.17 \pm 3.6 \%)$ and rotenone $(67.60 \pm 5.9 \%$ vs. $61.05 \pm 4.8 \%)$ (Figure 3A). Melatonin is unable to reverse the effect of etomoxir $(50.63 \pm 1.03 \%$ vs. $53.57 \pm 2.4 \%)$. Those oocytes were then activated parthenogenetically and cultured for further development. As shown in Figures 3B-D, melatonin promotes the embryo development indicated by the higher rate of blastocyst formation $(48.01 \pm 3.6 \%$ vs. $40.42 \pm 2.4)$. In contrast, the treatment of oocytes with etomoxir showed reduced two-cell embryo (72.90 $\pm 5.494 \%)$, four-cell embryo (66.47 $\pm 4.673 \%)$, and blastocyst (13.22 $\pm 0.02 .2 \%)$ formation rates, and melatonin could not correct these outcomes. Triacsin C-treated oocytes showed decreased four-cell embryo $(64.56 \pm 2.289 \%)$ and blastocyst $(19.28 \pm 2.48 \%)$ formation rates, which can be rescued by melatonin $(78.67 \pm 1.2 \%$ and 

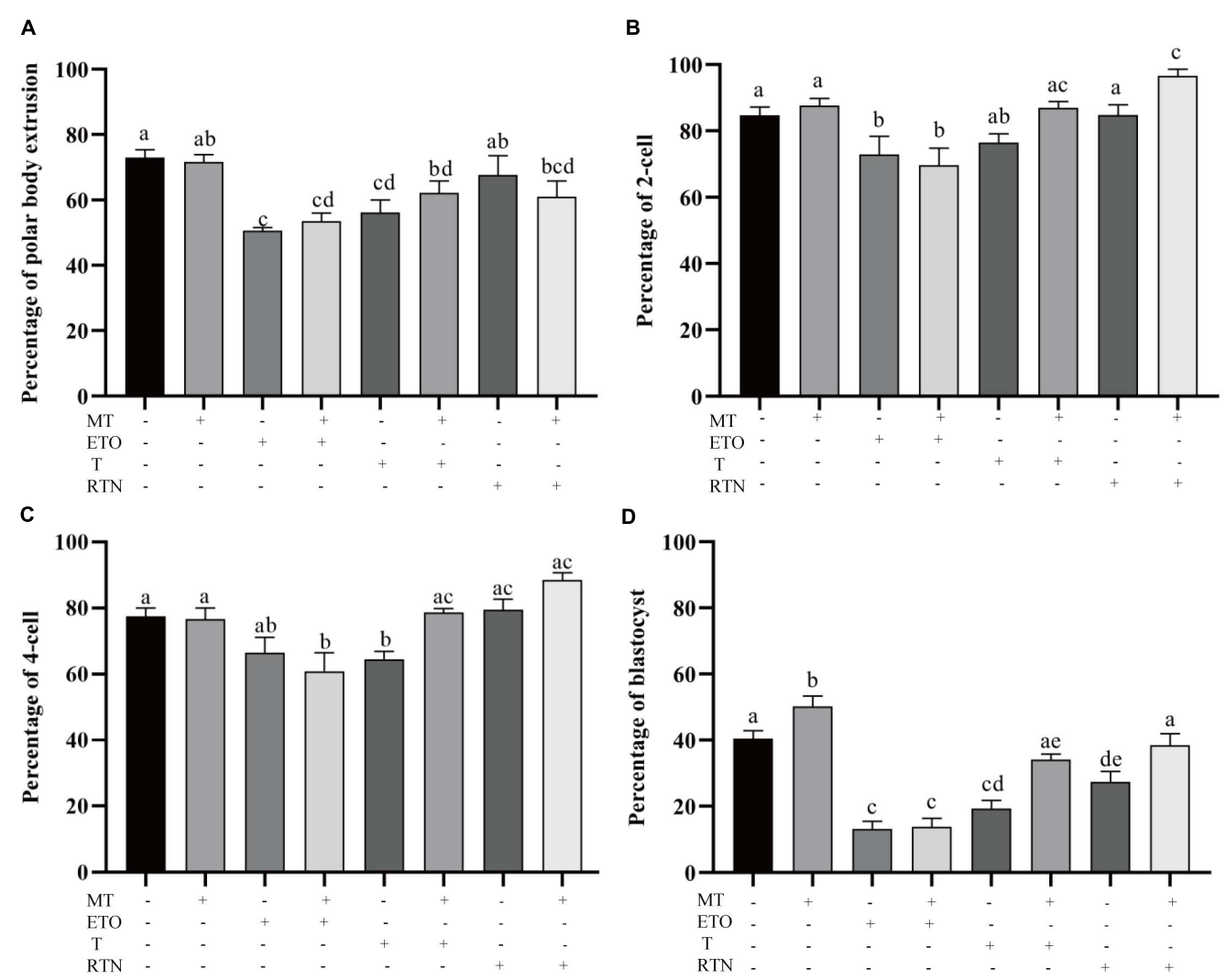

D

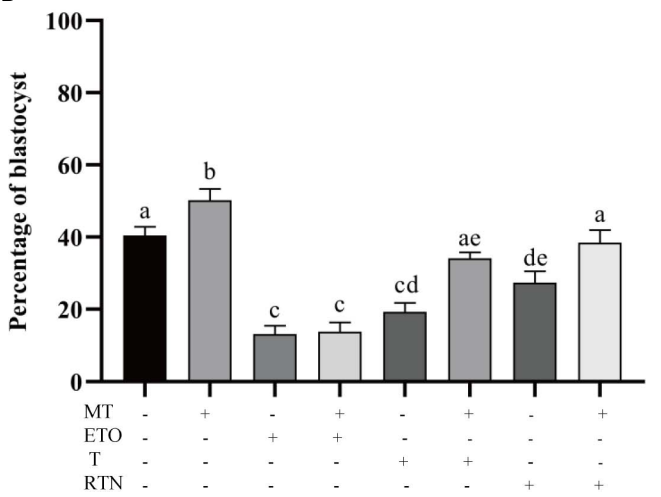

FIGURE 3 | Lipid metabolism regulates the developmental competence of porcine oocytes. (A) The percentage of polar body extrusion in porcine oocytes treated with control $(n=2,386)$, melatonin $(n=894)$, etomoxir $(n=1,303)$, etomoxir + melatonin $(n=1,301)$, triacsin $C(n=931)$, triacsin $C+$ melatonin $(n=1,026)$, rotenone $(n=710)$, and rotenone + melatonin $(n=706)$. (B) The percentage of two-cell embryos in porcine oocytes treated with control $(n=899)$, melatonin $(n=445)$, etomoxir $(n=739)$, etomoxir + melatonin $(n=654)$, triacsin C $(n=207)$, triacsin $C+$ melatonin $(n=315)$, rotenone $(n=414)$, and rotenone + melatonin $(n=328)$. (C) The percentage of four-cell embryos in porcine oocytes treated with control $(n=899)$, melatonin $(n=445)$, etomoxir $(n=739)$, etomoxir + melatonin $(n=654)$, triacsin C $(n=207)$, triacsin $C+$ melatonin $(n=315)$, rotenone $(n=414)$, and rotenone + melatonin $(n=328)$. (D) The percentage of blastocysts in porcine oocytes treated with control $(n=899)$, melatonin $(n=445)$, etomoxir $(n=739)$, etomoxir + melatonin $(n=654)$, triacsin $C(n=207)$, triacsin $C+$ melatonin $(n=315)$, rotenone $(n=414)$, and rotenone + melatonin $(n=328)$. Student's $t$-test and one-way ANOVA were utilized for statistical analyses. Different lowercase letters indicate significant differences. Error bars indicate SEM.

$34.12 \pm 1.7 \%$, respectively). Oocyte maturing in the medium containing rotenone showed a lower blastocyst formation rate $(27.40 \pm 3.1 \%)$, which can be corrected by melatonin $(38.46 \pm 0.3 .5 \%)$. The results presented here demonstrate that lipid metabolism is essential for porcine oocytes to acquire developmental competence and that melatonin suppresses lipid lyse and promotes lipid genesis, leading to enhanced developmental competence.

\section{Cumulus Cells Participate in the Regulation of Lipid Metabolism in Porcine Oocytes}

During the maturation process, the cumulus cells bidirectionally communicate with oocytes. Here, the transport of lipids from cumulus cells to oocytes was evaluated. Gap junction is pivotal for oocyte-cumulus cell communication. Thus, carbenoxolone (CBX), a gap junction blocker, was supplemented to the maturation medium, and the Lipi-Blue fluorescence intensity in oocytes was significantly deceased at $50 \mu \mathrm{M}(165.73 \pm 6.51)$ and $100 \mu \mathrm{M}(144.30 \pm 9.07)$. Meanwhile, the developmental competence of CBXtreated oocytes was also compromised (33.93 $\pm 2.7 \%$ vs. $47.70 \pm 2.3 \%)$. When oocytes were co-incubated with cumulus cells which were pre-stained with LipiBlue, as shown in Figure 4, the fluorescence intensity $(233.60 \pm 1.92)$ was significantly increased. Meanwhile, CBX-treated oocytes showed much lower fluorescence intensity $(79.90 \pm 5.43)$. These results indicate that the lipids are transferred from cumulus cells to oocytes through the gap junctions.

\section{Melatonin Receptors in Cumulus Cells Participate in the Regulation of Lipid Metabolism}

In order to investigate the mechanism underlying the beneficial effect of melatonin on oocyte maturation, the expression and function of its receptors (MT1/MT2) were evaluated. Firstly, luzindole-treated (the blocker for MT1/MT2) oocytes 


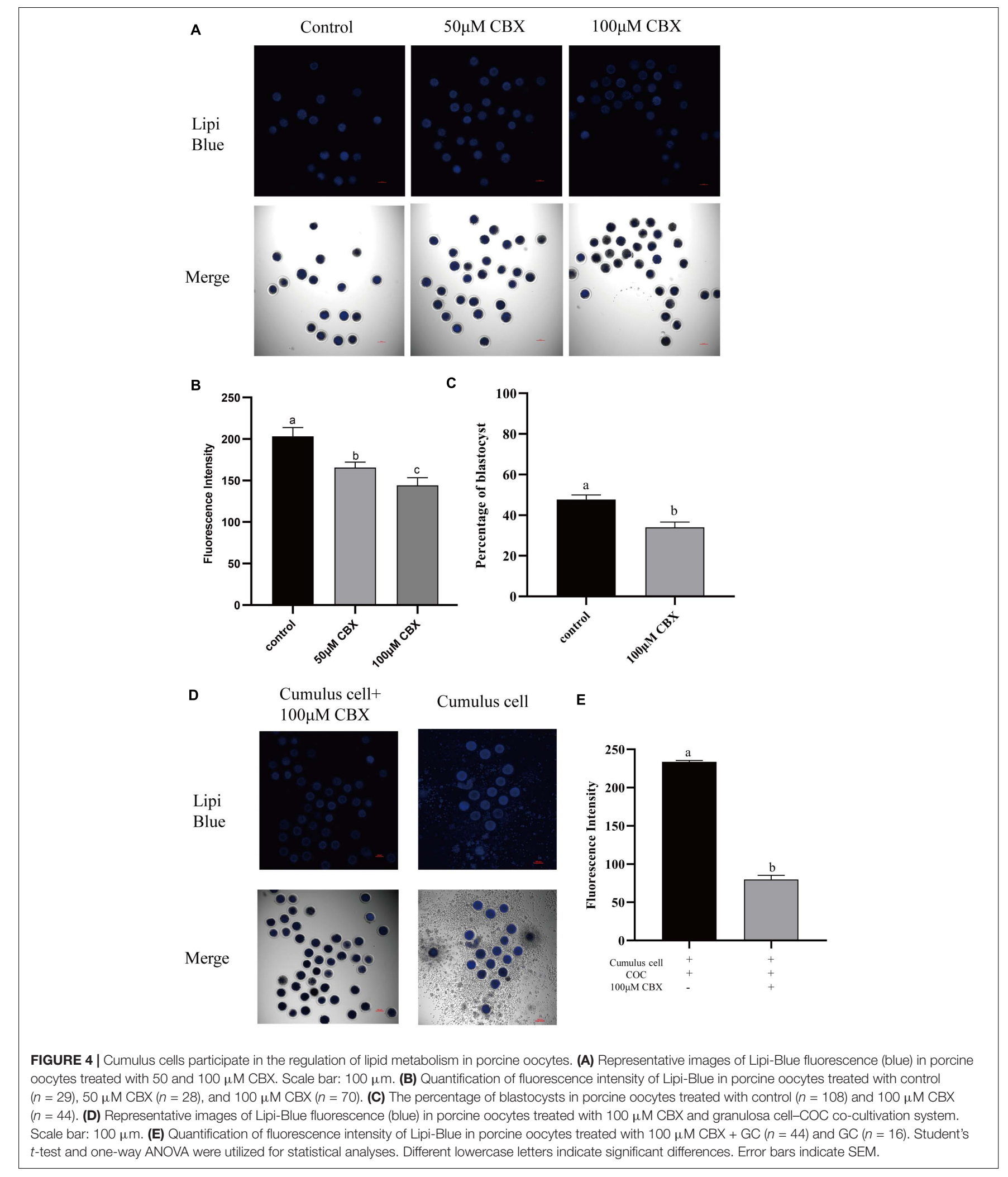

showed higher blue fluorescent intensity (155.25 \pm 5.3 ), which indicates the green fluorescent intensity was not altered $(163.21 \pm 6.077)$ (Figures 5A-D). The treatment with luzindole significantly reduced the porcine oocytes' ability to develop $(29.09 \pm 5.0 \%$ vs. $49.22 \pm 2.9 \%)$ (Figure 5E). Meanwhile, immunofluorescence with confocal microscopy observed that 


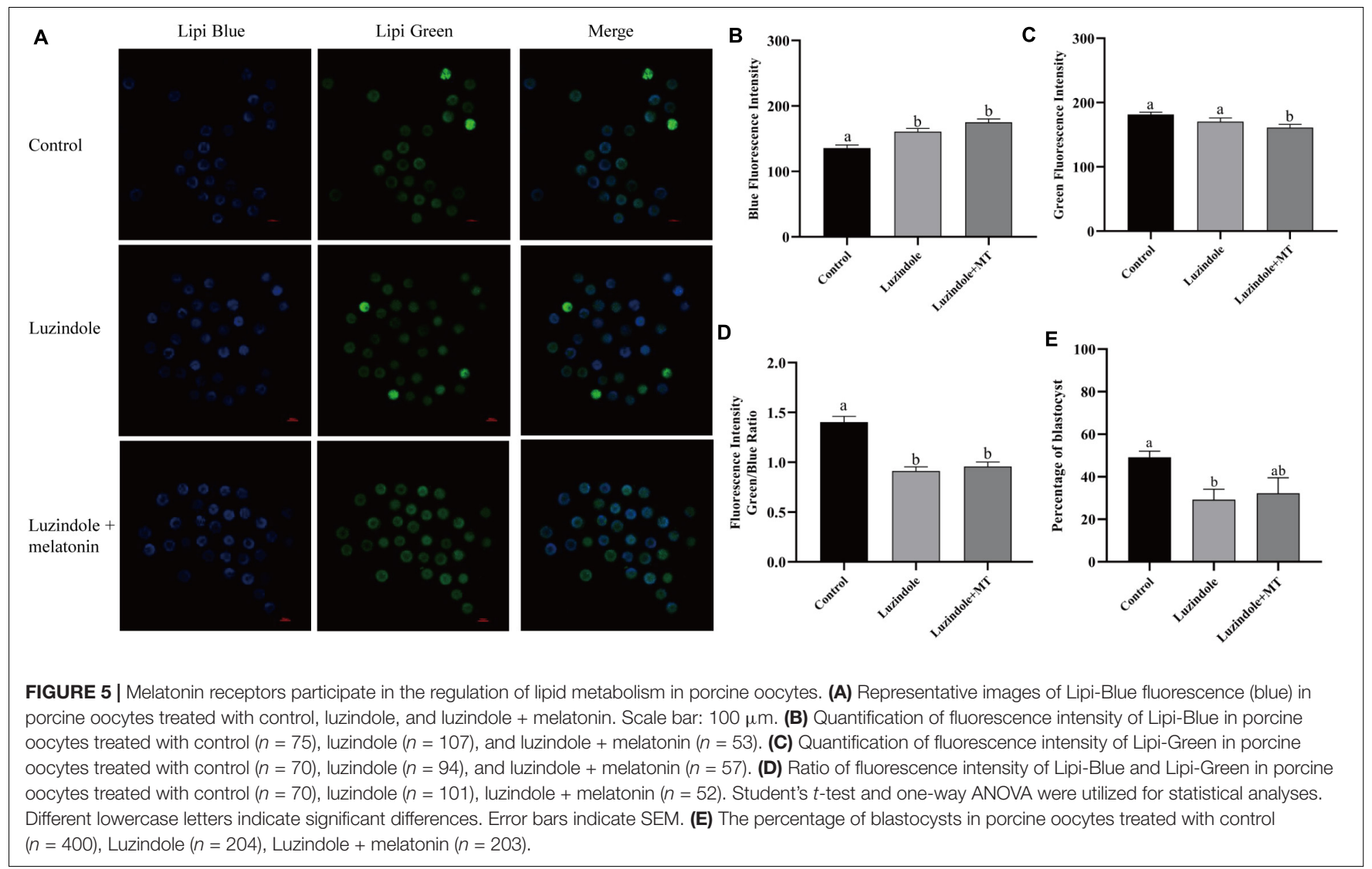

both melatonin receptors (MT1/MT2) were expressed in porcine cumulus cells (Figures 6A,B).

As shown in Figures 6C,D, the MII oocytes from melatonin receptor-knockout mice (Mtnrla $a^{-/}$and $M t n r 1 b^{-/-}$) were stained with Lipi-Blue, and the fluorescent intensity in oocytes from Mtnr1b ${ }^{-/-}(187.62 \pm 3.02)$ mice is lower than that from Mtnrla $a^{-/-}$and wild-type mice (203.17 \pm 2.3 and $199.31 \pm 3.27$, respectively). These results indicate melatonin receptors participate in the regulation of lipid genesis and transfer in cumulus cells.

\section{Melatonin Affects the Gene Expression in Porcine Cumulus Cells}

Transcriptomics analysis of cumulus cells demonstrated that melatonin induces upregulation of 1,528 genes, while 1,193 gene expressions are downregulated (fold change $>2$ or $<0.5$ ) (Figure $7 \mathbf{B}$ ). As the GO term demonstrated in Figures $7 \mathrm{C}-\mathrm{E}$, the top genes altered were related to antioxidants (SOD1, GPX1, GPX3, GPX4, PRDX2, and PRDX5), lipid metabolism (FABP3, FABP5, ACACB, TECR, etc.), and mitochondrial respiration (GPD1, ETFB, CYC1, ATP synthases, etc.). Genes related to lipid metabolism and mitochondrial biosynthesis were also evaluated by real-time q-PCR, but no differences were detected between cumulus cells from the control group and those from the melatonin group (Figure 7A).

\section{DISCUSSION}

Mammalian oocytes consume energy to achieve their final maturation such as germinal vesicle breakdown (GVBD), extrusion polar body, and redistribution of organelles. The basic pattern of energy production and utilization in mammalian oocytes has been recognized for more than half a century (Leese et al., 2016). It has been demonstrated that porcine oocytes are able to consume glucose and pyruvate to support their final maturation. However, in recent years, evidence that lipids are a pivotal nutrient for porcine or even the main energy source has accumulated (Bradley and Swann, 2019). In the current research, methods to monitor the dynamics of lipids in maturing porcine oocytes were established with Lipi-Blue and Lipi-Green probes. Together with lipid metabolism-related agonists, melatonin was found to suppress lipolysis but to increase lipid accumulation in porcine oocytes. Cumulus cells and melatonin receptors play a critical role in regulating oocyte lipid store for both pig and mouse, and melatonin targets the cumulus cells to promote oocyte maturation.

Porcine oocytes store exceptionally high amounts of endogenous lipid when compared with those in all other mammalian oocytes (McEvoy et al., 2000). In recent years, great progress had been made in elucidating the role of lipid metabolism in energy generation during oocyte maturation and early embryo development. Now, the morphological and biochemical changes in LDs can be considered important 


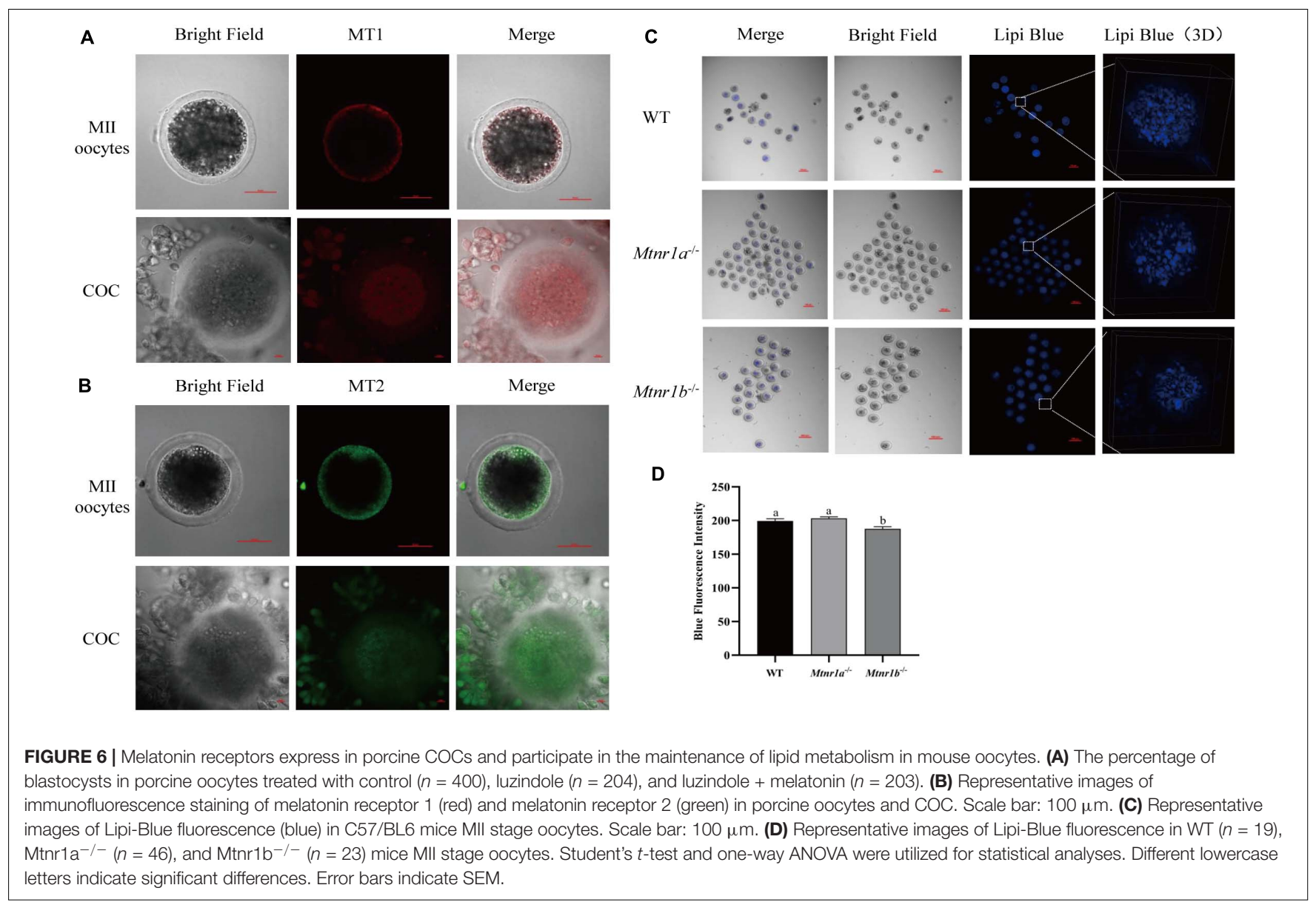

indicators of successful resumption of meiosis and maturation accomplishment. Here, we apply Lipi-Blue and Lipi-Green to detect the dynamics of LDs in live single oocytes. These probes are LD-specific fluorescent probes which allow monitoring of LDs in live cells even $48 \mathrm{~h}$ after staining and overcome the drawbacks of traditional dyes such as Nile Red and BODIPY 493/503 (Tatenaka et al., 2019). We successfully monitored the changes of lipids in live single porcine oocytes. The results confirmed that the lipid contents decreased as the oocytes went through the final maturation. Based on the decomposition of Nile Red spectra, researchers detected significant decreases in the level of triglycerides (17.7\%), phospholipids (26.4\%), and cholesterols $(23.9 \%)$ in porcine oocytes from immature to mature stages (Romek et al., 2011).

Meanwhile, previous studies found that oocytes with LDs centrally located showed a significantly higher rate of blastocyst development than the oocytes with LDs localized uniformly in the whole cytoplasm (Hiraga et al., 2013). In the current study, we observed that the LDs were accumulated in the center of porcine oocytes and that the treatment of etomoxir abolished the redistribution of LDs. Melatonin cannot correct the abnormal distribution of LDs. We used UPLC-MS/MS to analyze biochemical composition changes in oocytes. Another study using DESI-MS described differences in lipid composition among immature and in vitro mature porcine oocytes (Pirro et al., 2014). The disruption of the beta-oxidation pathway in mouse and cattle oocytes was found to negatively affect nuclear maturation and subsequent developmental potential (Ferguson and Leese, 2006; Dunning et al., 2010), and stimulation of FAO in mouse oocytes enhanced nuclear maturation (Dunning et al., 2011; Paczkowski et al., 2014). But the stimulation of lipolysis leads to inconsistent outcomes of oocyte nuclear and cytoplasmic maturation (Lowe et al., 2019). It was found that that stimulation of lipid metabolism by L-carnitine is beneficial to meiotic progression for porcine oocytes (Somfai et al., 2011; Wu et al., 2011), whereas another study showed that L-carnitine does not affect nuclear maturation (You et al., 2012). With the probes, it was observed that the disruption of lipolysis, lipid genesis, or mitochondrial activity all cause defects in oocyte maturation and development. It is evidenced that lipid metabolism is active throughout oocyte maturation and that melatonin modulates lipolysis and biogenesis to balance the energy supply and protect oocytes from ROS.

The beneficial effects of melatonin on mammalian oocytes have been well recognized. Evidence that melatonin enhances porcine oocyte maturation and embryo development has been accumulated, but the detailed mechanism is still unrevealed. Here, we present evidence to distinguish whether melatonin promotes porcine oocyte maturation through its receptors or its anti-oxidation capability. We observed that melatonin slows 
A

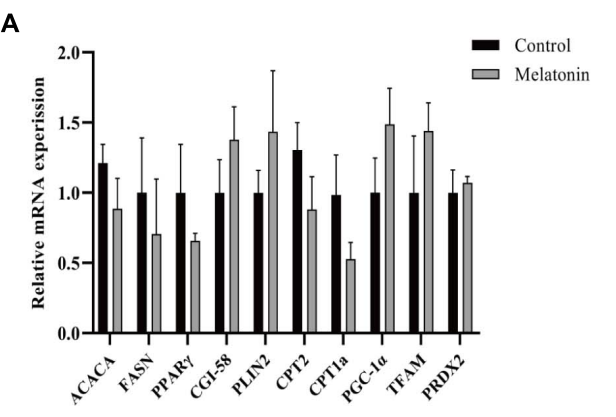

D

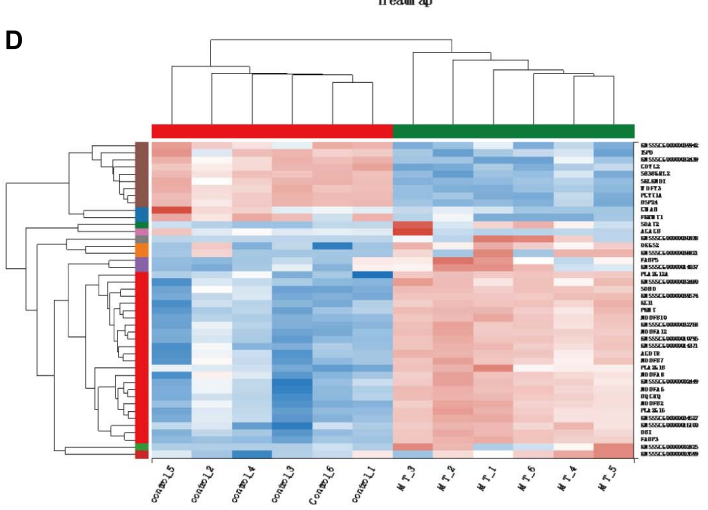

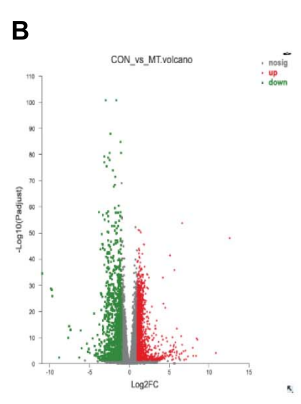
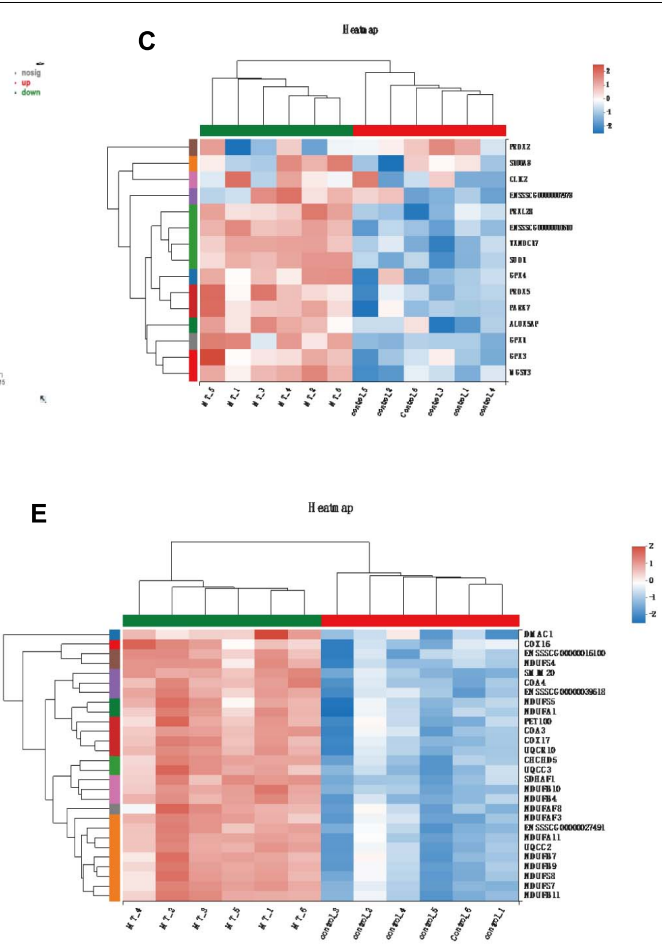

FIGURE 7 | Melatonin affects the gene expression in cumulus cells. (A) The mRNA levels of lipid metabolism and mitochondrial biosynthesis in porcine granulosa cells. Different lowercase letters indicate significant differences. Error bars indicate SEM. (B) The volcano plot showing upregulated (red) and downregulated (green) gene cumulus cells of the control and melatonin groups. (C-E) Heatmap showing average expression for all replicates and relative expression between replicates for genes with antioxidants, lipid metabolism, and mitochondrial respiration chain (based on GO terms).

down the mobilization of lipids stored in the oocytes, indicated by a lower fluorescence intensity at the MII stage than at the GV stage. In a previous study, it was claimed that oocytes treated with melatonin formed smaller LDs and increased the content of FAs, mitochondria number, and ATP level (Jin et al., 2017). But here, we found that melatonin suppresses lipolysis by evaluating the fluorescence intensity remaining in MII oocytes. However, another study showed that melatonin benefits porcine oocyte maturation by enhancing LD accumulation and triglyceride content in porcine oocytes (He et al., 2018). As we have observed, melatonin enhances the accumulation of lipids in oocytes. Together with the two previous researches, it can be speculated that melatonin balanced lipid biogenesis and utilization for the oocytes to generate sufficient ATP and limited ROS production to support the maturation of oocytes and subsequent embryonic development. Due to the lack of methods to observe ATP and ROS in live single cells, we are still not able to quantify the proper level of ATP/ROS for oocyte IVM.

Porcine oocytes are unable to accomplish their final maturation without the cumulus cell layer. Normally, the layers of cumulus cells surrounding ovulated oocytes directly have contact with the follicular fluid and bidirectionally transport small metabolites to regulate the growth and maturation of oocytes through gap junctions (Prates et al., 2014). In the current study, when the gap junction was disrupted by CBX, the oocytes showed lower amounts of lipids and compromised developmental competence. The cumulus cell layers play an important role in regulating lipid metabolism and transmitting the changes of free FA levels in the follicular fluid or culture medium. It had been observed that the cumulus cells functionally affect cytoplasmic mitochondrialipid distributions in porcine oocytes that matured in vitro and determine the acquisition of developmental competence (Cui et al., 2009). The latest research found that cumulus cells may balance FA accumulation in porcine oocytes, thus securing oocyte maturation. The supplementation of stearic acid in the maturation medium significantly increases the size and number of LDs in the cumulus cells but not those in oocytes; thus, it did not significantly alter the subsequent embryo development (Pawlak et al., 2020). When bovine oocytes are exposed to unsaturated or saturated FAs, the cumulus cells can store elevated FA in either the culture medium or follicle fluid to keep the ratio of saturated and unsaturated FAs in the proper range, thus protecting maturing oocytes from lipotoxicity (Aardema et al., 2011, 2013). The evidence presented here showed that cumulus cells directly participate in the regulation of the lipid store of oocytes. Blocking the gap junction could reduce the transport of lipids from cumulus cells to oocytes.

As we previously reported, melatonin receptors were identified on cumulus cells and oocytes of sheep and bovine (Tian et al., 2014, 2017; Wang et al., 2014). But for porcine oocytes, only RT-PCR analysis revealed the expression of the MT1 gene in cumulus and granulosa cells but not in oocytes 
(Kang et al., 2009). The MT2 receptor mediated the stimulatory effects of melatonin on porcine cumulus expansion and subsequent embryo development (Lee et al., 2018). MT1 and MT2 were expressed in porcine granulosa cells ( $\mathrm{He}$ et al., 2016). No research had been performed to investigate the role of melatonin on metabolism in cumulus cells. We found that the inhibitors of melatonin receptors remarkably reduced lipid mobilization and lipid biogenesis. Meanwhile, melatonin affects the expression of genes related to antioxidants, lipid metabolism, and mitochondrial respiration in cumulus cells. Altogether, melatonin receptors in cumulus cells play an essential role in conducting the regulatory signal induced by melatonin.

Our results provide a new perspective that reveals the mechanism of the beneficial effects of melatonin on porcine oocytes. With the methods to monitor lipid dynamics in maturing oocytes, it was confirmed that the lipid content decreases as maturation progresses. Melatonin may play an important role in balancing lipid biogenesis and utilization. It was observed that lipids are transferred from cumulus cells to oocytes through gap junctions and that melatonin receptors participate in this process. In summary, melatonin promotes oocyte maturation by regulating lipid metabolism through its receptors in cumulus cells. In the future, our results may be applied to clinical settings or the livestock industry where melatonin will be supplied to the diet for sows undergoing estrus or female undergoing ART treatment to help them generate high-quality oocytes.

\section{DATA AVAILABILITY STATEMENT}

The datasets presented in this study can be found in online repositories. The names of the repository/repositories and

\section{REFERENCES}

Aardema, H., Lolicato, F., van de Lest, C. H., Brouwers, J. F., Vaandrage, A. B., van Tol, H. T., et al. (2013). Bovine cumulus cells protect maturing oocytes from increased fatty acid levels by massive intracellular lipid storage. Biol. Reprod. 88:164. doi: 10.1095/biolreprod.112.106062

Aardema, H., Vos, P. L., Lolicato, F., Roelen, B. A., Knijn, H. M., Vaandrager, A. B., et al. (2011). Oleic acid prevents detrimental effects of saturated fatty acids on bovine oocyte developmental competence. Biol. Reprod. 85, 62-69. doi: 10.1095/biolreprod.110.088815

Abeydeera, L. R., Wang, W. H., Cantley, T. C., Rieke, A., Prather, R. S., and Day, B. N. (1998). Presence of epidermal growth factor during in vitro maturation of pig oocytes and embryo culture can modulate blastocyst development after in vitro fertilization. Mol. Reprod. Dev. 51, 395-401. doi: 10.1002/(SICI)10982795(199812)51:4<395::AID-MRD6>3.0.CO;2-Y

Aizawa, R., Ibayashi, M., Tatsumi, T., Yamamoto, A., Kokubo, T., Miyasaka, N., et al. (2019). Synthesis and maintenance of lipid droplets are essential for mouse preimplantation embryonic development. Development 146:dev181925. doi: $10.1242 /$ dev.181925

Bradley, J., and Swann, K. (2019). Mitochondria and lipid metabolism in mammalian oocytes and early embryos. Int. J. Dev. Biol. 63, 93-103. doi: 10. $1387 / \mathrm{ijdb} .180355 \mathrm{ks}$

Cetica, P., Pintos, L., Dalvit, G., and Beconi, M. (2002). Activity of key enzymes involved in glucose and triglyceride catabolism during bovine oocyte maturation in vitro. Reproduction 124, 675-681. doi: 10.1530/rep.0.1240675

Chen, L., Zhang, J. J., Zhang, X., Liu, X. L., Zhao, S. H., Huo, L. J., et al. (2020). Melatonin protects against defects induced by malathion during porcine oocyte maturation. J. Cell. Physiol. 235, 2836-2846. doi: 10.1002/jcp.29189 accession number(s) can be found in the article/supplementary material. The datasets generated for this study can be found in NCBI BioProject Accession ID PRJNA703733.

\section{ETHICS STATEMENT}

The animal study was reviewed and approved by the Animal Care and Use Committee of China Agricultural University.

\section{AUTHOR CONTRIBUTIONS}

LZ and GL proposed the research and revised the manuscript. TZ, SG, DL, MZ, LY, LS, and PJ performed the experiments. TZ and LZ analyzed the data and drafted the manuscript. All authors contributed to the article and approved the submitted version.

\section{FUNDING}

This study was supported by the National Natural Science Foundation of China (32002181 and 31830091) and Beijing Natural Science Foundation (6194040).

\section{ACKNOWLEDGMENTS}

We want to thank Ying Zhu and Xiufen Ye for assistance with the use of confocal microscopy.

Cheng, L. H., Qin, Y. S., Hu, X., Ren, L. K., Zhang, C., Wang, X. D., et al. (2019). Melatonin protects in vitro matured porcine oocytes from toxicity of Aflatoxin B1. J. Pineal Res. 66:e12543. doi: 10.1111/jpi.12543

Cui, M. S., Fan, Y. P., Wu, Y., Hao, Z. D., Liu, S., Chen, X. J., et al. (2009). Porcine cumulus cell influences ooplasmic mitochondria-lipid distributions, GSH-ATP contents and calcium release pattern after electro-activation. Theriogenology 71 , 412-421. doi: 10.1016/j.theriogenology.2008.08.008

Dunning, K. R., Akison, L. K., Russell, D. L., Norman, R. J., and Robker, R. L. (2011). Increased beta-oxidation and improved oocyte developmental competence in response to L-carnitine during ovarian in vitro follicle development in mice. Biol. Reprod. 85, 548-555. doi: 10.1095/biolreprod.110. 090415

Dunning, K. R., Cashman, K., Russell, D. L., Thompson, J. G., Norman, R. J., and Robker, R. L. (2010). Beta-oxidation is essential for mouse oocyte developmental competence and early embryo development. Biol. Reprod. 83, 909-918. doi: 10.1095/biolreprod.110.084145

Dunning, K. R., Russell, D. L., and Robker, R. L. (2014). Lipids and oocyte developmental competence: the role of fatty acids and $\beta$-oxidation. Reproduction 148, R15-R27. doi: 10.1530/REP-13-0251

Ferguson, E. M., and Leese, H. J. (1999). Triglyceride content of bovine oocytes and early embryos. J. Reprod. Fertil. 116, 373-378. doi: 10.1530/jrf.0.1160373

Ferguson, E. M., Leese, H. J. (2006). A potential role for triglyceride as an energy source during bovine oocyte maturation and early embryo development. Mol. Reprod. Dev. 73, 1195-1201. doi: 10.1002/mrd.20494

He, B., Yin, C., Gong, Y. B., Liu, J., Guo, H. D., and Zhao, R. Q. (2018). Melatonininduced increase of lipid droplets accumulation and in vitro maturation in porcine oocytes is mediated by mitochondrial quiescence. J. Cell. Physiol. 233, 302-312. doi: 10.1002/jcp.25876 
He, Y. M., Deng, H. H., Shi, M. H., Bodinga, B. M., Chen, H. L., Han, Z. S., et al. (2016). Melatonin modulates the functions of porcine granulosa cells via its membrane receptor MT2 in vitro. Anim. Reprod. Sci. 172, 164-172. doi: 10.1016/j.anireprosci.2016.07.015

Hiraga, K., Hoshino, Y., Tanemura, K., and Sato, E. (2013). Selection of in vitromatured porcine oocytes based on localization patterns of lipid droplets to evaluate developmental competence. J. Reprod. Dev. 59, 405-408. doi: 10.1262/ jrd.2012-126

Jin, J. X., Lee, S., Taweechaipaisankul, A., Kim, G. A., and Lee, B. C. (2017). Melatonin regulates lipid metabolism in porcine oocytes. J. Pineal Res. 62:e12388. doi: 10.1111/jpi.12388

Kang, J. T., Koo, O. J., Kwon, D. K., Park, H. J., Jang, G., Kang, S. K., et al. (2009). Effects of melatonin on in vitro maturation of porcine oocyte and expression of melatonin receptor RNA in cumulus and granulosa cells. J. Pineal Res. 46, 22-28. doi: 10.1111/j.1600-079X.2008.00602.x

Lee, S., Jin, J. X., Taweechaipaisankul, A., Kim, G. A., and Lee, B. C. (2018). Stimulatory effects of melatonin on porcine in vitro maturation are mediated by MT2 receptor. Int. J. Mol. Sci. 19:1581. doi: 10.3390/ijms1906 1581

Leese, H. J., Guerif, F., Allgar, V., Brison, D. R., Lundin, K., and Sturmey, R. G. (2016). Biological optimization, the Goldilocks principle, and how much is lagom in the preimplantation embryo. Mol. Reprod. Dev. 83, 748-754. doi: $10.1002 / \mathrm{mrd} .22684$

Li, B., and Dewey, C. N. (2011). RSEM: accurate transcript quantification from RNA-Seq data with or without a reference genome. BMC Bioinformatics 12:323. doi: 10.1186/1471-2105-12-323

Li, Y., Wang, J., Zhang, Z. Z., Yi, J. Y., He, C. J., Wang, F., et al. (2016). Resveratrol compares with melatonin in improving in vitro porcine oocyte maturation under heat stress. J. Anim. Sci. Biotechnol. 7:33. doi: 10.1186/s40104-0160093-9

Li, Y., Zhang, Z., He, C., Zhu, K., Xu, Z., Ma, T., et al. (2015). Melatonin protects porcine oocyte in vitro maturation from heat stress. J. Pineal Res. 59, 365-375. doi: $10.1111 /$ jpi.12268

Lowe, J. L., Bathgate, R., and Grupen, C. G. (2019). Effect of carbohydrates on lipid metabolism during porcine oocyte IVM. Reprod. Fertil. Dev. 31, 557-569. doi: $10.1071 /$ RD18043

Mateus, J. S., Tatiana D. S. R., Alessandra, T., Katia, R. A. B., Vanessa, G. S., Roniele, S. L., et al. (2016). Lipidome signatures in early bovine embryo development. Theriogenology 86, 472-484.e1. doi: 10.1016/j.theriogenology.2016.03.025

McEvoy, T. G., Coull, G. D., Broadbent, P. J., Hutchinson, J. S., and Speake, B. K. (2000). Fatty acid composition of lipids in immature cattle, pig and sheep oocytes with intact zona pellucida. J. Reprod. Fertil. 118, 163-170. doi: 10.1530/REPROD/118.1.163

Miao, Y., Zhou, C. Y., Bai, Q., Cui, Z. K., Xiayan, S. Y., Lu, Y., et al. (2018). The protective role of melatonin in porcine oocyte meiotic failure caused by the exposure to benzo(a)pyrene. Hum. Reprod. 33, 116-127. doi: 10.1093/humrep/ $\operatorname{dex} 331$

Niu, Y. J., Zhou, W., Nie, Z. W., Shin, K. T., and Cui, X. S. (2020). Melatonin enhances mitochondrial biogenesis and protects against rotenone-induced mitochondrial deficiency in early porcine embryos. J. Pineal Res. 68:e12627. doi: $10.1111 /$ jpi.12627

Paczkowski, M., Schoolcraft, W. B., and Krisher, R. L. (2014). Fatty acid metabolism during maturation affects glucose uptake and is essential to oocyte competence. Reproduction 148, 429-439. doi: 10.1530/REP-14-0015

Paczkowski, M., Silva, E., Schoolcraft, W. B., and Krisher, R. L. (2013). Comparative importance of fatty acid beta-oxidation to nuclear maturation, gene expression, and glucose metabolism in mouse, bovine, and porcine cumulus oocyte complexes. Biol. Reprod. 88:111. doi: 10.1095/biolreprod.113.108548

Park, H. J., Park, S. Y., Kim, J. W., Yang, S. G., Kim, M. J., Jegal, H. G., et al. (2018). Melatonin improves oocyte maturation and mitochondrial functions by reducing bisphenol A-derived superoxide in porcine oocytes in vitro. Int. J. Mol. Sci. 19:3422. doi: 10.3390/ijms19113422

Pawlak, P., Malyszka, N., Szczerbal, I., and Kolodziejski, P. (2020). Fatty acid induced lipolysis influences embryo development, gene expression and lipid droplet formation in the porcine cumulus cells. Biol. Reprod. 103, 36-48. doi: 10.1093/biolre/ioaa045

Pirro, V., Oliveri, P., Ferreira, C. R., González-Serrano, A. F., Machaty, Z., and Cooks, R. G. (2014). Lipid characterization of individual porcine oocytes by dual mode DESI-MS and data fusion. Anal. Chim. Acta 848, 51-60. doi: 10. 1016/j.aca.2014.08.001

Prates, E. G., Nunes, J. T., and Pereira, R. M. (2014). A role of lipid metabolism during cumulus-oocyte complex maturation: impact of lipid modulators to improve embryo production. Mediators Inflamm. 2014:692067. doi: 10.1155/ 2014/692067

Robinson, M. D., McCarthy, D. J., and Smyth, G. K. (2010). edgeR: a bioconductor package for differential expression analysis of digital gene expression data. Bioinformatics 26, 139-140. doi: 10.1093/bioinformatics/btp616

Romek, M., Gajda, B., Krzysztofowicz, E., Kepczynski, M., and Smorag, Z. (2011). New technique to quantify the lipid composition of lipid droplets in porcine oocytes and pre-implantation embryos using Nile Red fluorescent probe. Theriogenology 75, 42-54.

Shi, J. M., Tian, X. Z., Zhou, G. B., Wang, L., Gao, C., Zhu, S. E., et al. (2009). Melatonin exists in porcine follicular fluid and improves in vitro maturation and parthenogenetic development of porcine oocytes. J. Pineal. Res. 47, 318323. doi: 10.1111/j.1600-079X.2009.00717.x

Somfai, T., Kaneda, M., Akagi, S., Watanabe, S., Haraguchi, S., Mizutani, E., et al. (2011). Enhancement of lipid metabolism with L-carnitine during in vitro maturation improves nuclear maturation and cleavage ability of follicular porcine oocytes. Reprod. Fertil. Dev. 23, 912-920. doi: 10.1071/RD10339

Sturmey, R. G., and Leese, H. J. (2003). Energy metabolism in pig oocytes and early embryos. Reproduction 126, 197-204. doi: 10.1530/rep.0.1260197

Sudano, M. J., Rascado, T. D., Tata, A., Belaz, K. R., Santos, V. G., Valente, R. S., et al. (2016). Lipidome signatures in early bovine embryo development. Theriogenology 86, 472-484.e1.

Tatenaka, Y., Kato, H., Ishiyama, M., Sasamoto, K., Shiga, M., Nishitoh, H., et al. (2019). Monitoring lipid droplet dynamics in living cells by using fluorescent probes. Biochemistry 58, 499-503. doi: 10.1021/acs.biochem.8b01071

Tian, X. Z., Wang, F., He, C. J., Zhang, L., Tan, D. X., Reiter, R. J., et al. (2014). Beneficial effects of melatonin on bovine oocytes maturation: a mechanistic approach. J. Pineal Res. 57, 239-247. doi: 10.1111/jpi.12163

Tian, X. Z., Wang, F., Zhang, L., He, C. J., Ji, P. Y., Wang, J., et al. (2017). Beneficial effects of melatonin on the in vitro maturation of sheep oocytes and its relation to melatonin receptors. Int. J. Mol. Sci. 18:834. doi: 10.3390/ijms18040834

Trapnell, C., Pachter, L., and Salzberg, S. L. (2009). TopHat: discovering splice junctions with RNA-Seq. Bioinformatics 25, 1105-1111. doi: 10.1093/ bioinformatics/btp120

Wang, F., Tian, X. Z., Zhou, Y. H., Tan, D. X., Zhu, S. E., Dai, Y. P., et al. (2014). Melatonin improves the quality of in vitro produced (IVP) bovine embryos: implications for blastocyst development, cryotolerance, and modifications of relevant gene expression. PLoS One 9:e93641. doi: 10.1371/journal.pone. 0093641

Won, J. H., Park, S. J., Hong, S. J., Son, S. H., and Yu, J. W. (2015). Rotenoneinduced impairment of mitochondrial electron transport chain confers a selective priming signal for NLRP3 inflammasome activation. J. Biol. Chem. 290, 27425-27437. doi: 10.1074/jbc.M115.667063

Wu, G. Q., Jia, B. Y., Li, J. J., Fu, X. W., Zhou, G. B., Hou, Y. P., et al. (2011). L-Carnitine enhances oocyte maturation and development of parthenogenetic embryos in pigs. Theriogenology 76, 785-793. doi: 10.1016/J. THERIOGENOLOGY.2011.04.011

Xie, C., Mao, X. Z., Huang, J. J., Ding, Y., Wu, J. M., Dong, S., et al. (2011). KOBAS 2.0: a web server for annotation and identification of enriched pathways and diseases. Nucleic Acids Res. 39, W316-W322. doi: 10.1093/nar/ gkr483

You, J., Lee, J., Hyun, S. H., and Lee, E. (2012). L-Carnitine treatment during oocyte maturation improves in vitro development of cloned pig embryos by influencing intracellular glutathione synthesis and embryonic gene expression. Theriogenology 78, 235-243. doi: 10.1016/j.theriogenology.2012. 02.027

Yuan, Y., Spate, L. D., Redel, B. K., Tian, Y. C., Zhou, J., Prather, R. S., et al. (2017). Quadrupling efficiency in production of genetically modified pigs through improved oocyte maturation. Proc. Natl. Acad. Sci. U.S.A. 114, 5796-5804. doi: 10.1073/pnas.1703998114

Zhang, L., Zhang, Z. Z., Wang, J., Lv, D. Y., Zhu, T. Q., Wang, F., et al. (2019). Melatonin regulates the activities of ovary and delays the fertility decline in female animals via MT1/AMPK pathway. J. Pineal Res. 66:e12550. doi: 10.1111/ jpi. 12550 
Zhang, Y., Wang, T., Lan, M., Zang, X. W., Li, Y. L., Cui, X. S., et al. (2018). Melatonin protects oocytes from MEHP exposure-induced meiosis defects in porcine. Biol. Reprod. 98, 286-298. doi: 10.1093/biolre/iox185

Conflict of Interest: The authors declare that the research was conducted in the absence of any commercial or financial relationships that could be construed as a potential conflict of interest.
Copyright (c) 2021 Zhu, Guan, Lv, Zhao, Yan, Shi, Ji, Zhang and Liu. This is an open-access article distributed under the terms of the Creative Commons Attribution License (CC BY). The use, distribution or reproduction in other forums is permitted, provided the original author(s) and the copyright owner(s) are credited and that the original publication in this journal is cited, in accordance with accepted academic practice. No use, distribution or reproduction is permitted which does not comply with these terms. 